This is the author's version of a work that was accepted for publication in Electrochimica acta (Ed. Elsevier). Changes resulting from the publishing process, such as peer review, editing, corrections, structural formatting, and other quality control mechanisms may not be reflected in this document. Changes may have been made to this work since it was submitted for publication. A definitive version was subsequently published in:

Tsyntsaru, N. et al. "Toward uniform electrodeposition of magnetic Co-W mesowires arrays: direct versus pulse current depostion" in Electrochimica acta, vol. 188 (Jan. 2016), p. 589-601.

DOI 10.1016/j.electacta.2015.12.032€"

\title{
Toward uniform electrodeposition of magnetic Co-W mesowires arrays: direct versus pulse
}

\section{current deposition}

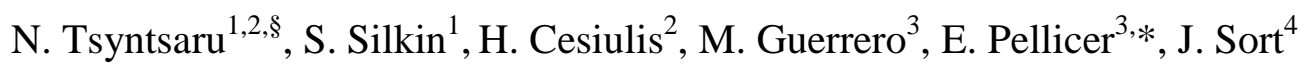

${ }^{1}$ Institute of Applied Physics of ASM, 5 Academy str., Chisinau, MD - 2028, Moldova

${ }^{2}$ Vilnius University, Dept. Phys. Chem., Naugarduko 24, Vilnius LT-03225, Lithuania

${ }^{3}$ Universitat Autònoma de Barcelona, Physics Department, E-08193 Bellaterra, Spain

${ }^{4}$ Institució Catalana de Recerca i Estudis Avançats (ICREA) and Universitat Autònoma de

Barcelona, Physics Department, E-08193 Bellaterra, Spain

${ }^{\S}$ ISE member

*Corresponding Author: Dr. Eva Pellicer (Eva.Pellicer@uab.cat) 


\begin{abstract}
Template-assisted electrodeposition of Co-rich Co-W alloys into mesoporous anodized aluminum oxide from ammonia-free solutions is investigated. The optimum deposition conditions rendering uniform filling of the pores, either by direct current (DC) or pulse current (PC) methods, are established. Contrary to the DC deposition on flat surfaces (which is kinetically controlled), the DC electrodeposition in the mesopores of the template is limited by mass transfer. Conversely, under PC mode, the Co-W electrodeposition process is controlled by kinetics at frequencies $>0.3 \mathrm{~Hz}$; and by diffusion at lower frequencies. The obtained mesowires are nanocrystalline and exhibit a hexagonal closed packed (hcp) structure. The magnetic properties of selected Co-W mesowires, with variable aspect ratio, are also studied. The arrays of mesowires exhibit a semi-hard ferromagnetic behavior with coercivity values that surpass those of Co mesowires with similar dimensions. The interplay between interwire dipolar interactions, magnetocrystalline anisotropy and shape anisotropy on the overall shape of the hysteresis loops (in particular, on the coercivity and squareness ratio values, as well as on the effective magnetic easy axis direction) is investigated in a semiquantitative manner.
\end{abstract}

Keywords: mesowires; direct current deposition; pulse current deposition; cobalt-tungsten alloy; magnetic properties. 


\section{Introduction}

Magnetic metallic wires have received a remarkable interest in the last few years due to their potential applications in devices such as tunable microwave filters [1,2], miniaturized magnetic sensors based on the giant magnetoresistance (GMR) effect [3-5] or magnetic recording media with perpendicular-to-plane effective anisotropy [6,7], among others. The implementation of efficient large-scale synthesis methods that allow the mass production of different types of metallic wires in a homogeneous and reproducible manner is essential to cope with the current technological needs in all the aforementioned areas. Electrodeposition inside porous templates is a rather convenient and industrially scalable technique for the fabrication of micro-, meso-, nano-structures, not only wires, but also rods or tubes. Advantages of electrodeposition over other synthetic methods (e.g. electrospinning or lithographic procedures) include: ease of operation and time-effectiveness, strict control of the wire length, precise tuning of the chemical composition and microstructure (i.e., crystallinity), and possibility to produce multilayered wires consisting of different segments with dissimilar compositions along the wire length [8].

For the growth of electrodeposited wires, either direct current or pulse plating conditions can be utilized. However, during direct current (or direct potential) electrodeposition, diffusion limitation effects can be quite prominent, hence causing a depletion of the metal ion concentration and/or a variation of $\mathrm{pH}$ at the bottom of the recesses. As a consequence, changes in the growing direction from nearly parallel to perpendicular to the wire axis, as well as changes in the crystal orientation of the wires can occur, thereby inducing variations in the resulting magnetic properties $[9,10]$. Pulse electrodeposition can be employed in order to minimize variations of $\mathrm{pH}$ and metal ion concentration in the course of electrodeposition, therefore overcoming the aforementioned problems. Furthermore, during electrodeposition at constant 
potential, simultaneously to the growth of the alloy in the recess, hydrogen evolves continuously and blocks the central part of the pores, thus shielding the deposition from the pore centre. Under these conditions, nanotubes are often formed. Conversely, in pulse potential mode, the evolved hydrogen has time to withdraw out of the pore during the pause time and the alloy can fill up the whole volume of the pore, resulting in the growth of fully dense wires.

The experimental conditions employed during pulse electrodeposition have a strong influence on the magnetic properties of the deposits [9]. Pulse plating has been satisfactorily used by several authors to fill all the pores of anodic aluminum oxide (AAO) templates with various metal nanowires (e.g., Ni or Co) [11,12]. During the study of Co [4-7,13-20], Ni [5,16, 19,21-23], and their alloys [24-26] it was concluded that the quality of the resulting wires strongly depends on the duty cycle. A relatively long pause between the pulses is preferable. Also, the magnetic behavior of nanowire arrays differs from that of continuous microcrystalline deposits. The wire aspect ratio and the interwire distance have an influence on the magnetic properties since both parameters control the magnetic dipolar interactions in the array $[4,6,7,15$ $18,27]$. Finally, in order to use nanowires/nanotubes in widespread devices, the materials should not only possess tunable magnetic properties but also acceptable wear and corrosion resistance. Also, the length of the electrodeposited wires/tubes must be uniform.

In this work, Co-W mesowires have been grown inside porous AAO templates (pore diameter $\sim 240 \mathrm{~nm}$ ), given the remarkable mechanical, magnetic and tribological properties of this alloy when deposited onto flat surfaces [28-30]. The possibility to fabricate Co-W nanostructures inside the pores of AAO from citrate-ammonia solutions was demonstrated in [31]. At constant potential mode, only tubes were typically obtained, whereas wires were formed during deposition using pulse potential mode. As aforementioned, this difference in the shape of the nanostructures can be understood by the different hydrogen evolution inside the recesses in 
each case. Moreover, problems were noticed to maintain a constant $\mathrm{pH}$ in ammonia-containing solutions during long-lasting electrodeposition at elevated temperatures [32].

The aim of the present study is two-fold. On the one hand, the template-assisted electrodeposition of Co-W alloys into mesoporous AAO from ammonia-free solutions is investigated, and the best conditions rendering uniform filling of the pores are established. A citrate bath for Co-W electrodeposition was selected based on successful results observed during deposition of Co-W alloys at $60{ }^{\circ} \mathrm{C}$ and $\mathrm{pH} 5.0,6.7$, and 8.0 on flat surfaces $[28,33]$ and wafers [34]. On the other hand, the magnetic properties of best-quality $\mathrm{Co}-\mathrm{W}$ mesowires, with variable aspect ratio, are also reported. The arrays of mesowires exhibit a semi-hard ferromagnetic behavior with coercivity values that surpass those of Co mesowires with similar dimensions.

\section{Experimental}

\subsection{Template-assisted deposition of mesowires}

Co-W mesowires were obtained by electrodeposition from a citrate electrolyte containing

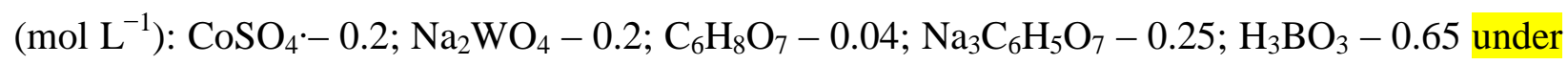
stagnant conditions. The $\mathrm{pH}$ was adjusted to $5.0,6.7$ and 8.0 by the addition of either $\mathrm{H}_{2} \mathrm{SO}_{4}$ or $\mathrm{NaOH}$. Bath temperature was maintained at 20,40 , or $60 \pm 2{ }^{\circ} \mathrm{C}$.

The electrodeposition was performed using an Autolab PGSTAT302N system with an $\mathrm{Ag} / \mathrm{AgCl}$ reference electrode. The counter electrode was a Pt mesh and the working electrode was a commercial Whatman AAO membrane with average pore-diameter of $240 \mathrm{~nm}$ and thickness of $60 \mu \mathrm{m}$. A Au layer was sputtered at the filter side to ensure the electrical contact ( $\mathrm{Au}$ sputtered layer thickness was $\sim 500 \mathrm{~nm}$ ). The working area was estimated taking into account $50 \%$ porosity of AAO template. 
Prior to electrodeposition, the working electrode was immersed in the electrolyte for several minutes under ultrasonic agitation in order to remove blocked gases and thereby improve electrolyte penetration into the pores. After the electrodeposition of Co-W mesowires, samples were rinsed with distilled water, ethanol and finally dried.

Co-W wire arrays were fabricated under: (i) potentiostatic mode at a constant cathodic potential $-E$ ranging from 0.8 to $1.1 \mathrm{~V}$; (ii) galvanostatic mode at a constant cathodic current density $-i$ from 6 to $12 \mathrm{~mA} \mathrm{~cm}^{-2}$; (iii) pulse current (PC) mode at pulse duration $\left(t_{o n}\right)$ from 4 to $2000 \mathrm{~ms}$, pulse cathodic current density $\left(-i_{\text {on }}\right)$ from 7.5 to $60 \mathrm{~mA} \mathrm{~cm}^{-2}$ and duty cycles $\gamma$ from 20 to $50 \%$, where $\gamma=\frac{t_{\text {on }}}{t_{\text {on }}+t_{\text {off }}} \cdot 100 \%$.

\subsection{Characterization of CoW mesowires' length, composition and structure}

Cross-section images were obtained using scanning electron microscopy (SEM) (Philips $\mathrm{XL30}$ ) at $30 \mathrm{kV}$, to obtain an enhanced contrast and have a clearer view of the length of the deposited alloy wires within the template. The cross-section SEM images might give the false impression that some of the pores remain unfilled and/or the wires are broken (i.e., they are not continuous). This stems from the fact that the AAO/CoW composite was manually broken so that the cut did not leave a flat surface underneath. Some wires or pieces of them were likely torn apart during breaking. The $\mathrm{W}$ content in the wires was determined by energy-dispersive X-ray (EDX) spectroscopy.

The uniformity of mesowires length in the arrays was estimated based on SEM crosssection images. The length deviation $(\Delta h)$ from average $\left(l_{\text {avg }}\right)$, maximum $\left(l_{\text {max }}\right)$ and minimum $\left(l_{\text {min }}\right)$ values of $\mathrm{Co}-\mathrm{W}$ wires length was determined as:

$$
\Delta h=\frac{\left(\left(l_{\max }-l_{\text {avg }}\right)+\left(l_{\text {avg }}-l_{\text {min }}\right)\right)}{2 \cdot l_{\text {avg }}} \cdot 100 \%
$$

The current efficiency $(C E)$ was calculated using the following expression: 


$$
\mathrm{CE}=\frac{H_{m}}{H_{t}} 100 \%
$$

where $H_{m}$ is the length of the mesowires measured from SEM images, $H_{t}$ is the theoretical length of the mesowires calculated using the Faraday's law under assumption that the overall density of the alloy additively comprises densities of pure components. Current density was calculated assuming a porosity percentage of $50 \%$.

Co-W mesowires were released from the AAO template in $10 \mathrm{wt} \% \mathrm{NaOH}$ at $80{ }^{\circ} \mathrm{C}$ during $5 \mathrm{~min}$ and rinsed afterwards in ethanol for transmission electron microscope (TEM) observations. Prior to imaging in TEM (Jeol-JEM 2011, $200 \mathrm{kV}$ ), the mesowires were dispersed onto a holey carbon-coated $\mathrm{Cu}$ support grid. Selected area electron diffraction (SAED) patterns were taken on different mesowires in order to determine the crystallographic structure.

\subsection{Magnetic characterization}

Hysteresis loops were acquired at room temperature using a vibrating sample magnetometer from Oxford Instruments, applying a maximum magnetic field of $10 \mathrm{kOe}$. The loops were measured on the mesowires still embedded in the AAO templates, applying the field both along and perpendicular to the wire long axis direction. Calculations of the average interwire dipolar fields were performed taking into account the mean mesowire dimensions and the interwire distance.

\section{Results and discussion}

\subsection{Study of electrochemical growth of Co-W mesowires}

\subsubsection{Potentiostatic/galvanostatic deposition under direct-current (DC) mode}


During galvanostatic electrodeposition, the current densities applied earlier $[33,34]$ for the growth of $\mathrm{Co}-\mathrm{W}$ on flat surfaces or into wafers having recesses of micrometric lateral sizes cannot be transferred "automatically" to the case of electrodeposition inside meso/nanopores. One cannot expect to obtain the same results because of at least two different reasons: (1) peculiarities of diffusion into the recesses at the mesoscale, and (2) the width of pores in industrial AAO membranes is uneven - smaller width at the bottom and larger toward the tophence resulting in a dynamic change of working area during the electrodeposition. The latter can be overcome to some extent by using potentiostatic rather than galvanostatic electrodeposition. Indeed, this mode has been used in the present study to determine the current densities that render uniform $\mathrm{Co}-\mathrm{W}$ mesowires.

Similarly to what was observed in micro-scaled wafers [34], electrodeposition inside the mesopores under potentiostatic conditions at $60{ }^{\circ} \mathrm{C}$, using relatively negative potentials and high $\mathrm{pH}(6.7$ or 8.0$)$, results in a strong influence of hydrogen evolution and concomitant alkalization at the electrode neighborhood. In these conditions, a non-uniform growth of $\mathrm{Co}-\mathrm{W}$ arrays of poor quality is obtained. Better uniformity in the mesowires length was achieved at $\mathrm{pH}=5$, when hydrogen evolution and alkalization of the near electrode space were minimized at the expense of decreasing the $\mathrm{W}$ content in the alloy. However, even at $\mathrm{pH}=5$, deposition carried out at high negative potential reveals abundant hydrogen evolution and low uniformity of the mesowire arrays (Fig. 1).

The chronoamperograms for $\mathrm{Co}-\mathrm{W}$ wire arrays deposited at $\mathrm{pH}=5$ and fixed low cathodic polarization of $-0.87 \mathrm{~V}$ are shown in Fig. 2(a) for different electrolyte temperatures. A decrease in temperature leads to less current fluctuations arising from hydrogen evolution. This favors higher uniformity of the electrodeposited Co-W arrays. Indeed, surveillance of length deviation (Fig. 2(b)) reveals that a lower electrolyte temperature assures not only lower hydrogen evolution, but also lower alloy deposition rates and, as a consequence, more uniform arrays of 
mesowires (Fig. 3). In all cases, the composition of the alloys was close to $\mathrm{Co}_{95} \mathrm{~W}_{5}$ at.\%, as determined by EDX.

Electrodeposition under galvanostatic control leads to the same tendency as for potentiostatic conditions, namely at higher current densities the growth is disturbed by hydrogen evolution. The best uniformity at $20^{\circ} \mathrm{C}$ is achieved at a cathodic current density of $-6 \mathrm{~mA} \mathrm{~cm}{ }^{-2}$, which roughly corresponds to potentiostatic electrodeposition at $-0.87 \mathrm{~V}$ (Fig. 4). Galvanostatic deposition at low current densities allows better control of Co-W electrodeposition. Note that the calculated apparent partial current density for metallic phase deposition obtained at $-12 \mathrm{~mA} \mathrm{~cm}{ }^{-2}$ is probably overestimated, because some pores were blocked by hydrogen and became inaccessible for electrodeposition.

As aforementioned, deposition proceeds in a more controlled manner only at relatively low cathodic potentials or current densities. This might be explained by peculiarities of diffusion inside the pores of the AAO template. Indeed, the presence of an array of cylindrical pores on the electrode has a significant effect on the diffusion [35]. When a metal is deposited inside nano- or mesopores, natural convection does not take place inside the pores due to the limited crosssection. Instead, metal ions are supplied by linear diffusion. Note that, regardless the mechanism for $\mathrm{Co}$ (II) discharge, all the forms of $\mathrm{Co}$ (II) complexes participate in the mass transfer. The most probable diffusion limitations encountered during $\mathrm{Co}-\mathrm{W}$ deposition in the pores were estimated using a simplified model of mass transfer in the pores.

The highest diffusion rate is to be observed when the concentration at the bottom surface of the pores with length $l$ is equal to zero: $C(t)_{x=0}=0$. In this case, diffusion undergoes in accordance with the second Fick's law. Under potentiostatic conditions, the concentration dependence on distance $(x)$ and time $(t)$ follows the Cottrell equation:

$$
C_{O}(x, t)=C_{O}^{*} \operatorname{erf}\left[\frac{x}{2 \sqrt{D_{0} t}}\right]
$$


where "erf" is an error function; $C_{0}{ }^{*}$ is the bulk concentration of metal ions; $D_{0}$ is the diffusion coefficient of metal complexes; and $t$ denotes the time.

Actually, $D_{0}$ values for specific metal complexes are not easily available and should be often estimated. Very small ligands (e.g., inorganic anions) replace water molecules in the inner hydration shell of the metal ion. The net difference between the $D_{0}$ values of the hydrated metal and the complex may be then rather small. Actually, they can be assumed to be equal, which is an acceptable approximation. Note that when the hydration shell is completely replaced by small hydrophobic anions like $\mathrm{Cl}^{-}$, the diffusion coefficient of the complex may become significantly greater than that of the hydrated ion. As the ligand size increases, the $D$ value of the complex tends toward that of the ligand [36]. For our simulations we used a value of apparent diffusion coefficient for Co citrate complexes of $7.9 \cdot 10^{-7} \mathrm{~cm}^{2} \mathrm{~s}^{-1}[37,38]$, i.e. the diffusion coefficient of cobalt due to complexation is 3 times lower than that reported in the literature for $\mathrm{Co}$ (II) in sulfate-ammonia solutions [39]. Also, the partial blocking of electrode surface by intermediates may have an influence on the apparent value of the diffusion coefficient [38].

In Fig. 5 the concentration $C(x, t)$ at $x=0.25 l, x=0.5 l, x=0.75 l$, and $x=l$ for $C_{0}{ }^{*}=0.20 \mathrm{M}$ as a function of time is shown. Although both $\mathrm{W}(\mathrm{VI})$ and Co(II) are present in the electrolyte, only $\mathrm{Co}$ (II) was considered because of the low tungsten content in the alloy. Indeed, considering the relatively low current densities used, partial hydrogen evolution current density is in all cases smaller than $-20 \mathrm{~mA} \mathrm{~cm}$. In these conditions, the thickness of the diffusion layer at $\mathrm{H}_{2-}$ evolving horizontal electrode is $40-80 \mu \mathrm{m}$ [40], which is comparable to the length of the pores. Therefore, the Nernst's diffusion layer concept and the Cottrell equation can be safely applied. The model reveals that the concentration gradient at the top of the pores is significant, i.e. $C\left(x=l, t_{x}\right)<C_{0}{ }^{*}$ already after 4 seconds from the beginning of the electrodeposition. Simultaneously, the maximum current density which can be supported by diffusion can be calculated by Cottrell's equation for current response: 


$$
i_{d}(t)=n F C_{O}^{*} \sqrt{\frac{D_{O}}{\pi t}}
$$

After 4 seconds diffusion from the bulk of solution toward the pores takes place and finally a stationary flux of metal ions can be settled:

$$
\left[\frac{\partial C_{O}(x, t)}{\partial x}\right]_{x=0}=\left[\frac{\partial C_{O}(x, t)}{\partial x}\right]_{x=l}
$$

and:

$$
i_{d}=\frac{n F C_{O}^{*} D_{O}}{l}
$$

The equation describing the time needed to switch from non-stationary to stationary diffusion $\left(t_{t r}\right)$ regimes can be derived from the equality between eq. (4) and (6):

$$
t_{t r}=\frac{l^{2}}{D_{O} \pi}
$$

In the studied case with pore length $l=60 \mu \mathrm{m}$, the value of $t_{t r}$ calculated from eq. (7) is $14.3 \mathrm{~s}$, and the diffusion current calculated using eq. (4) or (6) is equal to $5.1 \mathrm{~mA} \mathrm{~cm}{ }^{-2}$. This value is close to the one obtained at $20{ }^{\circ} \mathrm{C}$ on the experimental chronoamperograms shown in Fig. 2(a). This agreement indicates that the electrodeposition of $\mathrm{Co}-\mathrm{W}$ mesowires in our conditions is limited by mass transfer even at relatively low current densities. In other words, electrodeposition of the mesowires is governed by the diffusion of discharging metal-containing species from the bulk solution toward the pores, whereas Co and Co-W electrodeposition on flat electrodes proceeds under kinetic limitations [38]). This can be additionally proven by the current increase with temperature (see Fig. 2(a)), as higher activation energy holds under kinetic limitations in comparison with diffusion processes.

\subsubsection{Pulse current (PC) deposition}


Pulse deposition is often used to improve the control over the electrochemical process and, in turn, on the properties of electrodeposits such as morphology, crystallite size, chemical composition, crystalline structure and/or hydrogen inclusion [41-43]. By changing the frequency (or duty cycle) it is possible to provide either kinetic or diffusion (or mixed) control of electrode reaction. The kinetics of codeposition of $\mathrm{W}$ with $\mathrm{Co}$ (and other group metals) is rather complicated $[38,44]$ because of the adsorption of intermediates and solution components (e.g. ligands) on the electrode, coupled with charge transfer and diffusion processes. Therefore, it is impossible to distinguish in experimental electrochemical impedance (EIS) spectra, frequency ranges for which only the kinetics or the mass transfer control electrode processes apply $[38,45]$.

Simulation of EIS is a convenient way to estimate frequencies for which diffusion or kinetic limitations take place. In particular, diffusion gives rise to the occurrence of Warburg impedance. The simulation performed in this study was restricted to a Randles-Ershler equivalent circuit containing the following elements: solution resistance, double layer capacitance $\left(Q_{d l}\right)$, faradaic charge transfer resistance $\left(R_{c t}\right)$, and Warburg impedance. This impedance depends on the frequency of the potential perturbation and mass transfer conditions (concentrations, diffusion coefficients, and type of diffusion -semi-infinite or bounded diffusion). At high frequencies the Warburg impedance is small since diffusing reactants do not have to move very far; at low frequencies the reactants have to diffuse farther, thereby increasing the Warburg impedance. The typical shape of the Nyquist diagram for Randles-Ershler impedance is shown in Fig. 6(a). At a critical frequency $\left(\omega_{\text {crit }}\right)$ the control over the electrochemical reaction switches from kinetic to diffusion and vice versa.

For numerical simulations the values of circuit elements determined in [38] were used. A double layer capacitance of $1 \mu \mathrm{F} \mathrm{cm} \mathrm{cm}^{-2}(\mathrm{n}=0.85)$ was employed. To calculate the Warburg constant, the diffusion of main components in citrate solution at $\mathrm{pH}=5$ were considered: $\mathrm{CoHCitr}^{-}$(as oxidant) and all forms of citrates $\mathrm{HCitr}^{-}$(as reductant). Taking into account that $\left[\mathrm{Co}^{2+}\right]=0.20 \mathrm{M}$, the concentration of all the forms of citrate in the diffusion layer is $0.20 \mathrm{M}$ [44]. 
Their diffusion coefficient was taken as $D_{\text {citr }}=5.910^{-6} \mathrm{~cm}^{2} \mathrm{~s}^{-1}$ [46], thus the Warburg coefficient is equal to $\sigma=0.3614 \Omega \mathrm{cm}^{2} \mathrm{~s}^{-1}$. Finally, the values of $R_{c t}$ depend on the deposition potential and vary from $6544 \Omega \mathrm{cm}^{2}$ (at low polarization, i.e., in the range from -0.70 to -0.55 V) to $226 \Omega \mathrm{cm}^{2}$ (at high polarization, e.g., $-0.86 \mathrm{~V}$ ). The calculated dependence of charge transfer resistance $\left(R_{c t}\right)$ on the values of $\omega_{c r i t}$ is presented in the insert of Fig. 6(a). Numerical simulation revealed that the values of $\omega_{c r i t}$ depend mainly on the values of $R_{c t}$ and $\sigma$. Namely, the higher the charge transfer resistance, the lower the value of $\omega_{\text {crit }}$, i.e. the transition from kinetics control to diffusion control occurs at lower frequencies. In our Co-W electrolyte, $\omega_{\text {crit }} \leq 0.3 \mathrm{~Hz}$ in all cases. This means that at higher frequencies the electrode processes are controlled by charge transfer rate, whereas at lower frequencies diffusion is governing the process. Figure 6(b) and (c) display the EIS spectra in Nyquist and Bode coordinates, respectively, recorded during electrodeposition of $\mathrm{Co}-\mathrm{W}$ at $\mathrm{pH} 6.7$ and $E=-0.8 \mathrm{~V}$ inside mesopores of $200 \mathrm{~nm}$ in diameter. The experimental spectra are described by an equivalent circuit (inset of Figure 6(c)) containing an adsorption stage. Elements take the following values: R1=9.83 $\Omega \mathrm{cm}^{2} ; \mathrm{R} 2=535 \Omega \mathrm{cm}^{2}$; CPE1 $=0.0021 \mathrm{~F} \mathrm{~cm}^{-2}(\mathrm{n}=0.63) ; \mathrm{CPE} 2=0.00715 \mathrm{~F} \mathrm{~cm}^{-2}(\mathrm{n}=0.60) ; \mathrm{R} 3=104 \Omega \mathrm{cm}^{2}$. The large capacitance values are probably caused by the formation of unevenly distributed oxidecontaining intermediates on the surface.

For electrodeposition in PC mode, pulse durations of $4 \mathrm{~ms}, 20 \mathrm{~ms}, 200 \mathrm{~ms}$, and $2000 \mathrm{~ms}$ were applied. These correspond to sinusoidal frequencies of $125 \mathrm{~Hz}, 25 \mathrm{~Hz}, 2.5 \mathrm{~Hz}$, and $0.25 \mathrm{~Hz}$, respectively, for a duty cycle of $50 \%$. Taking into account that the concentration gradient decreases during the rest time, it is possible to state that the electrode process is controlled by the kinetics of charge transfer reaction even at the longest pulse duration applied (2000 ms).

Electrodeposition at pulse cathodic current densities $\left(i_{\text {on }}\right)$ within the range of $-i=7.5-60$ $\mathrm{mA} \mathrm{cm}{ }^{-2}$ and at a duty cycle of $20-50 \%$ was investigated. The characteristic influence of pulse duration on the uniformity of mesowires growth is shown in Fig. 7. The most uniform growth 
was obtained for the shortest pulse duration (4 ms). Likewise, the effect of duty cycle is shown in Fig. 8. Uniformity worsens at high duty cycles (i.e. $50 \%$ and $33 \%$ ) probably because the pause duration is exceedingly short to allow the hydrogen bubbles to be released from the template while a new portion of electrolyte penetrates inside the pores from the bulk of the solution. In addition, mass transport limitation is neglected at shorter pulses and smaller duty cycles (equivalent to high frequencies). It should be mentioned that with increasing the pause duration mesowires grow in a more uniform manner, but the total duration of electrodeposition to obtain the same length of mesowires also increases. In addition, for a large pulse current density and pulse duration, the average current density exceeds the limiting diffusion current, which is equal to $\sim 5 \mathrm{~mA} \mathrm{~cm}^{-2}$ under our deposition conditions, composition of solution, and geometry of pores (see section 3.1.1). Therefore, in this case hydrogen evolution dominates.

Furthermore the duty cycle has an impact on the tungsten content along the wires (i.e., compositional homogeneity) (Fig. 9). For a given current density, at lower duty cycle (which means longer pause duration) the tungsten content along the mesowires is much more homogeneous.

\subsection{Structural characterization of Co-W mesowires}

Figure 10 shows representative TEM images of Co-W wires deposited by DC and PC conditions. The mesowires were of nanocrystalline nature and exhibited the hexagonal closepacked (hcp) structure, independently of the electrodeposition conditions and final wire length (see SAED patterns in Figs. 10b and 10d). This was to some extent expected given the low W content (below 6 at.\%) in all the wires (Figure 9). It is known that amorphization in electrodeposited Co-W films typically takes place for higher W contents (> 30 at.\%) [33]. Also it is known that the face-centered cubic (fcc) phase of Co is promoted during electrodeposition of nanowires only for rather acid $\mathrm{pH}$ values, close to $\mathrm{pH} 2$ [12, 47], which is not the case of our 
study. Finally, according to the Co-W binary phase diagram, $\mathrm{W}$ is also stabilizer of the hcp phase when dissolved in small amounts into Co [48].

Remarkably, although the conditions corresponding to the mesowire in Figure 10(a) were the best ones within the DC regime, residual porosity was noticed both by TEM and SEM. This porosity stems for the hydrogen co-evolution that accompanies metal ions discharge. Meanwhile, wires obtained by PC deposition looked more compact. The measured lengths were in agreement with the values previously determined by SEM.

\subsection{Magnetic properties}

Hysteresis loops were recorded on selected arrays of mesowires, still embedded in the AAO templates, prepared under both direct current and pulse plating conditions. The selected samples correspond to deposition at $\mathrm{pH}=5$ and $20{ }^{\circ} \mathrm{C}$, with optimum conditions to attain homogeneous filling of the pores. Two different sets of mesowires were grown by DC and PC, with different lengths, to compare the properties of short (about $1 \mu \mathrm{m}$ long) versus long (about $25 \mu \mathrm{m}$ long) mesowires.

Representative hysteresis loops, measured along and perpendicular to the mesowire axes, are shown in Figure 11. The values of coercivity, $H_{C}$, for the different investigated samples are listed in Table 1. Since all samples are polycrystalline (hcp structure) and contain a similar W content (3-5 at.\%), it is unlikely that the slight variations in microstructure/composition can explain the observed changes in $H_{C}$. Table 1 reveals that, in fact, $H_{C}$ decreases when the length of the mesowires is increased. Such inverse relationship between $H_{C}$ and sample/particle size is typical of ferromagnetic materials and has been observed also in other metallic nanowires systems [49]. In ferromagnetic alloys, $H_{C}$ usually increases when the particle size is reduced due to a transition from a multi-domain to a mono-domain magnetic configuration, where only coherent rotation of the magnetic moments (but not propagation of domain walls) takes place 
during magnetization reversal. However, no pronounced changes in the domain structure are anticipated between the two sets of short or long mesowires. Actually, in a first approximation, the mesowires can be considered to behave as magnetic dipoles. In arrays of nanowires, the existence of magnetic dipolar interactions between the wires has been reported to have a net effect on $H_{C}$ [50]. Typically, $H_{C}$ decreases with an increase of magnetic dipolar interactions. These interactions mainly depend on the mesowires aspect ratio and the average interwire distance. Moreover, $H_{C}$ is larger when measured along the wires axis $(/ /)$, as compared to hysteresis loops recorded along the perpendicular-to-the-wires axis $(\perp)$. This can be ascribed to the trade-off between the effective shape anisotropy of the mesowires and the net role of interwire dipolar interactions. It is remarkable that the measured values of $H_{C}$ in the Co-W mesowires are larger than those of pure fcc-Co or hcp-Co for wires with similar diameter and aspect ratio and interwire distance, where $H_{C}$ typically ranges between 100 and 250 Oe $[27,47,49,50]$.

Isolated polycrystalline mesowires with a large aspect ratio should exhibit a square hysteresis loop when the magnetic field is applied parallel to the wires axis and a tilted hard-axis loop along the perpendicular direction, due to the shape anisotropy [50-53]. Conversely, the investigated arrays of $\mathrm{Co}-\mathrm{W}$ wires exhibit sheared hysteresis loops along both directions of measurement. Such behavior can be ascribed to the existence of interwire magnetic dipolar interactions; more specifically, to the competition between shape anisotropy, magnetocrystalline anisotropy and the dipolar fields created between neighboring wires. Figure 11 reveals that for the shortest mesowires, the magnetic easy axis in the array remains the one along the mesowire axis, where slightly larger $M_{R} / M_{S}$ and $H_{C}$ values are obtained with respect to the measurements acquired applying the field along the perpendicular-to-the-wire direction. Conversely, such a trend is lost for the long mesowires, where the loops along both directions of measurement virtually overlap. 
For a two-dimensional infinite array of cylindrical particles with radius $r$, length $L$, aspect ratio $A_{r}=\mathrm{L} / 2 \mathrm{r}$ and interwire distance $D$, the total dipolar field created when all wires are oriented along the wire axis direction can be written as $[27,49,51]$ :

$$
\mathrm{H}_{\mathrm{dip}, 0^{\circ}}=\frac{4.2 \mathrm{M}_{\mathrm{S}} \pi \mathrm{r}^{2} \mathrm{~L}}{\mathrm{D}^{3}}
$$

If all moments are aligned perpendicular to the wires direction, then the dipolar field would be:

$$
\mathrm{H}_{\mathrm{dip}, 90^{\circ}}=\frac{-2.1 \mathrm{M}_{\mathrm{S}} \pi \mathrm{r}^{2} \mathrm{~L}}{\mathrm{D}^{3}}
$$

In turn, the self-demagnetizing field of one wire can be expressed as:

$$
\mathrm{H}_{\text {demag }}=\left(\mathrm{N}_{\perp}-\mathrm{N}_{\|}\right) \mathrm{M}_{\mathrm{S}}
$$

where $\mathrm{N}_{\|}$and $\mathrm{N}_{\perp}$ are the demagnetizing factors along the wire axis and its perpendicular direction and can be calculated using a prolate spheroid approximation, as follows [54]:

$$
\begin{gathered}
\mathrm{N}_{\|}=\frac{4 \pi}{\mathrm{A}_{\mathrm{r}}^{2}-1}\left[\frac{\mathrm{A}_{\mathrm{r}}}{\left.2 \sqrt{\left(\mathrm{A}_{\mathrm{r}}^{2}-1\right)} \ln \left(\mathrm{A}_{\mathrm{r}}+\sqrt{\mathrm{A}_{\mathrm{r}}^{2}-1}\right)-1\right]}\right. \\
\mathrm{N}_{\perp}=\frac{4 \pi-\mathrm{N}_{\|}}{2}
\end{gathered}
$$

Hence, the total effective anisotropy field can be then expressed as: 


$$
\mathrm{H}_{\mathrm{K}, \text { eff }}=\left(\mathrm{N}_{\perp}-\mathrm{N}_{\|}\right) \mathrm{M}_{\mathrm{S}}-\frac{6.3 \mathrm{M}_{\mathrm{S}} \pi \mathrm{r}^{2} \mathrm{~L}}{\mathrm{D}^{3}}+\mathrm{H}_{\mathrm{mc}}
$$

Here $H_{m c}$ is the magnetocrystalline anisotropy contribution, which for single-crystalline hcp-Co is negative and is approximately equal to $5 \mathrm{M}_{\mathrm{S}}$ [27]; for polycrystalline mesowires, $H_{m c}$ averages out and is close to 0 . Equation 13 predicts that if $H_{K, \text { eff }}$ is positive the magnetic easy axis will be $\|$ to the wires axis; otherwise, for negative $H_{K, \text { eff }}$ the magnetic easy axis will tend to be oriented along the $\perp$ direction [49]. If one uses approximate values of: wire length, wire diameter and distance between wires (pitch size), one can try to estimate the effective anisotropy field of a given array of mesowires. Alternatively, one can determine a critical mesowire length, $L_{C}$ that would render $H_{K, \text { eff }}=0$, i.e., the critical length beyond which the effective anisotropy direction would switch from the $\|$ to the $\perp$ directions. Considering that $\left(\mathrm{N}_{\perp}-\mathrm{N}_{\|}\right) \approx 2 \pi, r=120 \mathrm{~nm}, D=$ $450 \mathrm{~nm}$ (as estimated from SEM images of the AAO template) and that our material is mainly hcp-Co (i.e., $0<\mathrm{H}_{\mathrm{mc}}<5 \mathrm{M}_{\mathrm{S}}$, depending on the degree of polycrystallinity), Equation 13 then predicts that $\mathrm{L}_{\mathrm{C}}$ will range from approximately $0.5 \mu \mathrm{m}$ to $2 \mu \mathrm{m}$. Hence, this simplified reasoning provides a semi-quantitative explanation of why the effective easy axis along the $\|$ direction in the arrays of short mesowires is lost for the case of the longer mesowires.

\section{Conclusions}

The main conclusions of the present study can be summarized as follows:

1. The best filling of AAO mesopores by $\mathrm{Co}-\mathrm{W}$ alloy was obtained at room temperature using $\mathrm{pH}=5$ : in DC mode at low current densities or polarizations; in PC mode at shorter pulse durations and smaller duty cycles, i.e. under conditions at which the influence of diffusion is minimized. 
2. Under direct current or potential mode the electrode process undergoes under kinetic control on flat surfaces whereas the process is limited by mass transfer during electrodeposition in the mesopores. For the membranes used in this work (pore length about $60 \mu \mathrm{m}$, pore diameter $240 \mathrm{~nm}$ ) the following diffusion processes were revealed: after $4 \mathrm{~s}$ from the beginning of electrodeposition the diffusion from the bulk of the solution toward the pores starts and after $\sim 14 \mathrm{~s}$ diffusion evolves from non-stationary to stationary. Under PC mode the Co-W electrodeposition process is controlled by kinetics at frequencies $>0.3 \mathrm{~Hz}$; and by diffusion at lower frequencies.

3. The magnetic properties of selected arrays of mesowires (the ones grown under optimum conditions for homogeneous filling of the pores) reveal a semi-hard ferromagnetic behavior and a strong influence of interwire magnetic dipolar interactions on the resulting shape of the hysteresis loops. While a clear magnetic easy direction along the mesowires long axis is observed for short mesowires, such a trend is lost for longer mesowires. The interplay between shape anisotropy, magnetocrystalline anisotropy and the dipolar fields created between neighboring wires can explain this observation.

\section{Acknowledgements}

This work has been partially funded by the MIP-031/2014 project from the Research Council of Lithuania, Moldavian projects 14.02.121A and 14.819.02.16F, the 2014-SGR-1015 project from the Generalitat de Catalunya, the MAT2014-57960-C3-1-R from the Spanish Ministerio de Economía y Competitividad (MINECO) and the MANAQA FET-Open Project from the European Commission. Dr. Eva Pellicer is grateful to MINECO for the "Ramon y Cajal" contract (RYC-2012-10839). M. Guerrero acknowledges the support of the Secretary for Universities and Research of the Government of Catalonia and the COFUND Programme of the 
Marie Curie Actions of the 7th R\&D Framework Programme of the European Union for the 'Beatriu de Pinos' contract (2013 BP-B 00077). 


\section{References}

[1] G.A. Ozin, Nanochemistry: Synthesis in diminishing dimensions, Adv. Mater. 4 (1992) 612-649.

[2] C.R. Martin, Nanomaterials: a membrane-based synthetic approach, Science 266 (1994) 1961-1966.

[3] A. Fert, L. Piraux, Magnetic nanowires, J. Magn. Magn. Mater. 200 (1999) 338-358.

[4] G. Dumpich, T.P. Krome, B. Hausmanns, Magnetoresistance of single Co nanowires, J. Magn. Magn. Mater. 248 (2002) 241-247.

[5] X.W. Wang, Z.H. Yuan, J.S. Li, (110) Orientation growth of magnetic metal nanowires with face-centered cubic structure using template synthesis technique, Mater. Charact. 62 (2011) $642-646$.

[6] Y. Ren, J. Wang, Q. Liu, B. Zhang, X. Han, D. Xue, Abnormal coercivity dependence on the diameter of Co nanowires in anodic aluminium oxide templates, J. Phys. D: Appl. Phys. 42 (2009) 105002-105006.

[7] Y. Yang, Y. Chen, Y. Wu, X. Chen, M. Kong, Diameter-controllable magnetic properties of Co nanowire arrays by pulsed electrodeposition, J. Nanomater. 2012 (2010) 793854-793858.

[8] G.D. Sulka, Highly ordered anodic porous alumina formation by self-organized anodizing, in: A. Eftekhari (Ed.), Nanostructured materials in electrochemistry, Wiley-VCH Verlag GmbH \& Co. KGaA, Weinheim, Germany, 2008.

[9] M.A. Kashi, A. Ramazani, Z. Fallah, Magnetic properties improvement through off time between pulses and annealing in pulse electrodeposited CoZn nanowires, J. Alloy. Compd. 509 (2011) 8845-8849.

[10] M. Darques, L. Piraux, A. Encinas, P. Bayle-Guillemaud, A. Popa, U. Ebels, Electrochemical control and selection of the structural and magnetic properties of cobalt nanowires, Appl. Phys. Lett. 86 (2005) 072508-072511. 
[11] K. Nielsch, F. Müller, A.P. Li, U. Gösele, Uniform nickel deposition into ordered alumina pores by pulsed electrodeposition, Adv. Mater. 12 (2000) 582-586.

[12] B. Jang, E. Pellicer, M. Guerrero, X. Chen, H. Choi, B.J. Nelson, J. Sort, S. Pané, Fabrication of segmented $\mathrm{Au} / \mathrm{Co} / \mathrm{Au}$ nanowires: insights in the quality of $\mathrm{Co} / \mathrm{Au}$ junctions, $\mathrm{ACS}$ Appl. Mater. Interfaces 6 (2014) 14583-14589.

[13] Y.P. Ivanov, L.G. Vivas, A. Asenjo, A. Chuvilin, O. Chubykalo-Fesenko, M. Vázquez, Magnetic structure of a single-crystal hcp electrodeposited cobalt nanowire, EPL 102 (2013) $17009-17014$.

[14] B. Hamrakulov, I.-S. Kim, M.G. Lee, B.H. Park, Electrodeposited Ni, Fe, Co and Cu single and multilayer nanowire arrays on anodic aluminum oxide template, Trans. Nonferrous Met. Soc. China 19 (2009) s83-s87.

[15] D. Reuter, S. Hoch, B. Hausmanns, B. Stahlmecke, G. Dumpich, R. Wieser, U. Nowak, A.D. Wieck, Hall magnetometry on Co, Fe, and Py nanowires, J. Magn. Magn. Mater. 272-276 (2004) 1654-1655.

[16] P.M. Paulus, F. Luis, M. Kröll, G. Schmid, L.J. de Jongh, Low-temperature study of the magnetization reversal and magnetic anisotropy of $\mathrm{Fe}, \mathrm{Ni}$, and $\mathrm{Co}$ nanowires, J. Magn. Magn. Mater. 224 (2001) 180-196.

[17] H. Zeng, M. Zheng, R. Skomski, D.J. Sellmyer, Y. Liu, L. Menon, S. Bandyopadhyay, Magnetic properties of self-assembled Co nanowires of varying length and diameter, J. Appl. Phys. 87 (2000) 4718-4721.

[18] X.H. Huang, G.H. Li, X.C. Dou, L. Li, Magnetic properties of single crystalline Co nanowire arrays with different diameters and orientations, J. Appl. Phys. 105 (2009) 084306084310.

[19] J. Sellmyer, M. Zheng, R. Skomski, Magnetism of Fe, Co and Ni nanowires in selfassembled arrays, J. Phys.: Condens. Matter 13 (2001) R433-R460. 
[20] S. Pal, S. Saha, D. Polley, A. Barman, Magnetization reversal dynamics in Co nanowires with competing magnetic anisotropies, Solid State Commun. 151 (2011) 1994-1998.

[21] C.-L. Xu, H. Li, G.-Y. Zhao, H.-L. Li, Electrodeposition of ferromagnetic nanowire arrays on $\mathrm{AAO} / \mathrm{Ti} / \mathrm{Si}$ substrate for ultrahigh-density magnetic storage devices, Mater. Lett. 60 (2006) 2335-2338.

[22] S. Thongmee, H.L. Pang, J. Ding, J.Y. Lin, Fabrication and magnetic properties of metallic nanowires via AAO templates, J. Magn. Magn. Mater. 321 (2009) 2712-2716.

[23] M. Kröll, W.J. Blau, D. Grandjean, R.E. Benfield, F. Luis, P.M. Paulus, L.J. de Jongh, Magnetic properties of ferromagnetic nanowires embedded in nanoporous alumina membranes, J. Magn. Magn. Mater. 249 (2002) 241-245.

[24] J. Fu, S. Cherevko, C.-H. Chung, Electroplating of metal nanotubes and nanowires in a high aspect-ratio nanotemplate, Electrochem. Commun. 10 (2008) 514-518.

[25] L. Belliard, J. Miltat, A. Thiaville, S. Dubois, J.L. Duvail, L. Piraux, Observing magnetic nanowires by means of magnetic force microscopy, J. Magn. Magn. Mater. 190 (1998) 1-16.

[26] H.R. Khan, K. Petrikowski, Synthesis and properties of the arrays of magnetic nanowires of Co and CoFe, Mater. Sci. Eng. C-Mater. Biol. Appl. 19 (2002) 345-348.

[27] J. Rivas, A.K.M. Bantu, G. Zaragoza, M.C. Blanco, M.A. López-Quintela, Preparation and magnetic behavior of arrays of electrodeposited Co nanowires, J. Magn. Magn. Mater. 249 (2002) 220-227.

[28] N. Tsyntsaru, S. Belevsky, A. Dikusar, J.-P. Celis, Tribological behavior of electrodeposited cobalt-tungsten coatings: dependence on current parameters, Trans. Inst. Metal Finish. 86 (2008) 301-307.

[29] N. Tsyntsaru, A. Dikusar, H. Cesiulis, J.-P.Celis, Z. Bobanova, S. Sidelnikova, S. Belevskii, Y. Yapontseva, O. Bersirova, V. Kublanovskii, Tribological and corrosive characteristics of electrochemical coatings based on cobalt and iron superalloys, Powder Metall. Met. Ceram. 48 (2009) 419-428. 
[30] P. Bera, H. Seenivasan, K.S. Rajam, C. Shivakumara, S.K. Parida, Characterization and microhardness of $\mathrm{Co}-\mathrm{W}$ coatings electrodeposited at different $\mathrm{pH}$ using gluconate bath: A comparative study, Surf. Interface Anal. 45 (2013) 1026-1036.

[31] H. Cesiulis, X.G. Xie, E. Podlaha-Murphy, Electrodeposition of Co-W alloys with P and Ni, Mater. Sci.-Medzg. 15 (2009) 115-122.

[32] H. Cesiulis, E.J. Podlaha-Murphy, Electrolyte considerations of electrodeposited Ni-W alloys for microdevice fabrication, Mater. Sci.-Medzg. 9 (2003) 324-327.

[33] N. Tsyntsaru, H. Cesiulis, E. Pellicer, J.-P. Celis, J. Sort, Structural, magnetic, and mechanical properties of electrodeposited cobalt-tungsten alloys: intrinsic and extrinsic interdependencies, Electrochim. Acta 104 (2013) 94-103.

[34] N. Tsyntsaru, G. Kaziukaitis, C. Yang, H. Cesiulis, H.G. Philipsen, M. Lelis, J.-P. Celis, Co-W nanocrystalline electrodeposits as barrier for interconnects, J. Solid State Electrochem. 18 (2014) 3057-3064.

[35] M.C. Lopes, C.P. de Oliveira, E.C. Pereira, Computational modeling of the templateassisted deposition of nanowires, Electrochim. Acta 53 (2008) 4359-4369.

[36] J. Bufle, Z. Zhang, K. Startchev, Metal flux and dynamic speciation at (bio)interfaces, Environ. Sci. Technol. 47 (2007) 7609-7620.

[37] T. Cohen-Hyams, Electrodeposition of thin metallic magnetic films, Thesis, Tamuz 5763 Haifa, 2003.

[38] S.S. Belevskii, H. Cesiulis, N.I. Tsyntsaru, A.I. Dikusar, The role of mass transfer in the formation of the composition and structure of $\mathrm{CoW}$ coatings electrodeposited from citrate solutions, Surf. Eng. Appl. Electrochem. 46 (2010) 570-578.

[39] C.H. Rios-Reyes, M. Granados-Neri, L.M. Mendoza-Huizar, Kinetic study of the cobalt electrodeposition onto glassy carbon electrode from ammonium sulfate solutions, Quim. Nova 32 (2009) 2382-2386. 
[40] C.A.C. Sequeira, D.M.F. Santos, B. Šljukic; L. Amaral, Physics of electrolytic gas evolution, Braz. J. Phys., 43 (2013) 199-208

[41] F.H. Su, C.S. Liu, P. Huang, Friction and wear of nanocrystalline Co and Co-W alloy coatings produced by pulse reverse electrodeposition, Wear 30 (2013) 114-125.

[42] F.-H. Su, C.-S. Liu, P. Huang, Effect of complexing agents and $\mathrm{pH}$ on microstructure and tribological properties of Co-W coatings produced by double pulse electrodeposition, Appl. Surf. Sci. 258 (2012) 6550-6557.

[43] M. Mulukutla, V.K. Kommineni, S.P. Harimkar, Pulsed electrodeposition of Co-W amorphous and crystalline coatings, Appl. Surf. Sci. 258 (2012) 2886-2893.

[44] N. Tsyntsaru, H. Cesiulis, M. Donten, J. Sort, E. Pellicer, E.J. Podlaha-Murphy, Modern trends in tungsten alloys electrodeposition with iron group metals, Surf. Eng. Appl. Electrochem. 48 (2012) 491-520.

[45] Y. Liu, W. Wang, Investigation on the $\mathrm{Cu}(\mathrm{II})$ and $\mathrm{Co}(\mathrm{II})$ electrochemical reduction process in citrate solution by CV and EIS, J. Electrochem. Soc. 159 (2012) D375-D381.

[46] M.Z. Southard, L.J. Dias, K.J. Himmelstein, V.J. Stella, Experimental determinations of diffusion coefficients in dilute aqueous solutions using the method of hydrodynamic stability, Pharm. Res. 8 (1991) 1489-1494.

[47] J.U. Cho, J.-H. Wu, J.H. Min, S.P. Ko, J.Y. Soh, Q.X. Liu, Y.K. Kim, Control of magnetic anisotropy of Co nanowires, J. Magn. Magn. Mater. 303 (2006) e281-e285.

[48] Binary alloy phase diagrams, T. B. Massalski, H. Okamoto (Eds.), $2^{\text {nd }}$ Edition, ASM International, 1990.

[49] G.C. Han, B.Y. Zong, P. Luo, Y.H. Wu, Angular dependence of the coercivity and remanence of ferromagneticnanowire arrays, J. Appl. Phys. 93 (2003) 9202-9207.

[50] V. Raposo, J.M. Garcia, J.M. González, M. Vázquez, Long-range magnetostatic interactions in arrays of nanowires, J. Magn. Magn. Mater. 222 (2000) 227-232. 
[51] D.J. Sellmyer, M. Zheng, R. Skomski, Magnetism of Fe, Co and Ni nanowires in selfassembled arrays, J. Phys.: Condens. Matter 13 (2001) R433-R460.

[52] J. Qin, J. Nogués, M. Mikhaylova, A. Roig, J.S. Muñoz, M. Muhammed, Differences in the magnetic properties of $\mathrm{Co}, \mathrm{Fe}$, and $\mathrm{Ni} 250-300 \mathrm{~nm}$ wide nanowires electrodeposited in amorphous anodized alumina templates, Chem. Mater. 17 (2005) 1829-1834.

[53] M. Grimsditch, Y. Jaccard, I.K. Schuller, Magnetic anisotropies in dot arrays: Shape anisotropy versus coupling, Phys. Rev. B 58 (1998) 11539-11543.

[54] M. Vázquez, L.G. Vivas, Magnetization reversal in Co-base nanowire arrays, Phys. Status Solidi B 248 (2011) 2368-2381. 


\section{Figure captions}

Figure 1. (a) Chronoamperograms of $\mathrm{Co}-\mathrm{W}$ mesowires deposition into $\mathrm{AAO}$ at $\mathrm{pH}=5$ and 60 ${ }^{\circ} \mathrm{C}$ and SEM cross-section images corresponding to (b) $-1.0 \mathrm{~V}$ and $2880 \mathrm{~s}$ and (c) $-0.9 \mathrm{~V}$ and $2667 \mathrm{~s}$.

Figure 2. (a) Chronoamperograms of $\mathrm{Co}-\mathrm{W}$ mesowires deposition into $\mathrm{AAO}$ at $\mathrm{pH}=5, E=-$ $0.87 \mathrm{~V}$, and different temperatures. (b) Thickness deviation $(\Delta \mathrm{h})$ and deposition rate (DR) as a function of temperature.

Figure 3. SEM cross-section images of Co-W mesowires obtained by potentiostatic deposition at $-0.87 \mathrm{~V}, \mathrm{pH}=5$ and temperature: (a) $20^{\circ} \mathrm{C}$, (b) $40^{\circ} \mathrm{C}$, and (c) $60^{\circ} \mathrm{C}$.

Figure 4. SEM cross-section images of Co-W mesowires obtained by $\mathrm{DC}$ at $\mathrm{pH}=5$ and $20{ }^{\circ} \mathrm{C}$ under the following cathodic current densities and deposition times: (a) $-6 \mathrm{~mA} \mathrm{~cm}{ }^{-2}, 21600 \mathrm{~s}$; (b) $-7.5 \mathrm{~mA} \mathrm{~cm}^{-2}, 21600 \mathrm{~s}$; (c) $-12 \mathrm{~mA} \mathrm{~cm}^{-2}, 7200 \mathrm{~s}$. The inset in (a) is a zoomed detail of a few mesowires (scale bar $400 \mathrm{~nm}$ ).

Figure 5. Concentration of metal ions, i.e. $\mathrm{Co}(\mathrm{II})$, as a function of time at various distances (x) from the bottom of the pores, as calculated using eq. (3); $l=60 \mu \mathrm{m}, D_{0}=7.9 \times 10^{-7} \mathrm{~cm}^{2} \mathrm{~s}^{-1}$, $C_{0}{ }^{*}=0.20 \mathrm{M}$.

Figure 6. (a) Nyquist diagram for Randles circuit including semi-infinite linear diffusion. Insert presents the calculated dependence of $\omega_{\text {crit }}$ on charge transfer resistance $\left(R_{c t}\right)$. Values of circuit elements are indicated in the text. Experimental (open circles) and fitted (solid line) EIS spectra in (b) Nyquist and (c) Bode coordinates recorded during Co-W electrodeposition at $\mathrm{pH} 6.7$ and $E$ $=-0.8 \mathrm{~V}$ inside mesopores of $200 \mathrm{~nm}$ in diameter. Inset in (c) is the equivalent circuit. R1 is uncompensated resistance; R2 is charge transfer resistance; CPE1 is constant phase element of 
double electric layer; R3 and CPE2 are adsorption resistance and constant phase element (capacity), respectively.

Figure 7. Pulse duration effect. SEM cross-section images of Co-W mesowires obtained under PC deposition at $\mathrm{pH}=5,20{ }^{\circ} \mathrm{C}$, duty cycle of $20 \%$, and different $t_{\text {on }}$ times. Deposition time was $28800 \mathrm{~s}$ at $i_{\text {on }}=-15 \mathrm{~mA} \mathrm{~cm}^{-2}$ and $7200 \mathrm{~s}$ at $i_{\text {on }}=-60 \mathrm{~mA} \mathrm{~cm}^{-2}$.

Figure 8. Duty cycle effect. SEM images of Co-W mesowires obtained by $\mathrm{PC}$ deposition at $\mathrm{pH}$ $=5,20^{\circ} \mathrm{C}, i_{\text {on }}=-15 \mathrm{~mA} \mathrm{~cm}{ }^{-2}, \mathrm{t}_{\mathrm{on}}=4 \mathrm{~ms}$, and different duty cycle and deposition time: (a) 50\%, $11632 \mathrm{~s}$; (b) 33\%, $17448 \mathrm{~s}$; and (c) 20\%, $28800 \mathrm{~s}$.

Figure 9. Dependence of tungsten content in $\mathrm{Co}-\mathrm{W}$ wires as a function of the duty cycle at $\mathrm{pH}=$ $5,20{ }^{\circ} \mathrm{C}, i_{\text {on }}=-15 \mathrm{~mA} \mathrm{~cm}^{-2}$, and $t_{\text {on }}=4 \mathrm{~ms}$.

Figure 10. (a) TEM image of the end of a mesowire and (b) corresponding SAED pattern for Co-W wires obtained by DC mode at $-6 \mathrm{~mA} \mathrm{~cm}^{-2}$ for $14400 \mathrm{~s}\left(\mathrm{pH}=5,20^{\circ} \mathrm{C}\right)$. (c) TEM image of a bundle of Co-W wires obtained by PC deposition at $i_{o n}=-15 \mathrm{~mA} \mathrm{~cm}{ }^{-2}, t_{o n}=4 \mathrm{~ms}$. The insets in (a) and (c) show a less magnified view of the wire and the bottom part of a wire showing the typical branched structure, respectively. The overall length of the mesowires is $40 \mu \mathrm{m}$ in both cases.

Figure 11. Hysteresis loops of selected arrays of mesowires (embedded in the AAO templates), grown by DC (a,b) and PC (c,d) conditions, applying the external magnetic field along and perpendicular to the wires direction. Panels (a) and (c) correspond to short mesowires $(\sim 1 \mu \mathrm{m}$ long) whereas panels (b) and (d) show the loops of longer mesowires ( $25 \mu \mathrm{m}$ long). 
Table 1. Summary of the magnetic properties of Co-W wire arrays

\begin{tabular}{|c|c|c|c|c|c|}
\hline Current mode & $\begin{array}{c}\text { Wires length, } \\
\mu \mathrm{m}\end{array}$ & $\begin{array}{l}\mathbf{H}_{\mathrm{C}} \perp \\
(\mathbf{O e})\end{array}$ & $\begin{array}{l}\mathbf{H}_{\mathrm{C}} \| \\
(\mathbf{O e})\end{array}$ & $\begin{array}{c}\mathbf{M}_{\mathbf{R}} / \mathbf{M}_{\mathbf{S}} \\
\perp\end{array}$ & $\begin{array}{c}\mathbf{M}_{\mathbf{R}} / \mathbf{M}_{\mathbf{S}} \\
\|\end{array}$ \\
\hline $\mathrm{DC},-6 \mathrm{~mA} \mathrm{~cm}{ }^{-2}$, short & 1.2 & 499 & 499 & 0.21 & 0.28 \\
\hline $\begin{array}{c}\mathrm{PC}, i_{\text {on }}=-15 \mathrm{~mA} \mathrm{~cm}^{-2}, t_{\text {on }}=4 \\
\mathrm{~ms}, \text { short }\end{array}$ & 0.8 & 510 & 730 & 0.20 & 0.23 \\
\hline $\mathrm{DC},-6 \mathrm{~mA} \mathrm{~cm}^{-2}$, long & 26.0 & 264 & 234 & 0.08 & 0.07 \\
\hline $\begin{array}{c}\mathrm{PC}, i_{\text {on }}=- \\
\mathrm{ms}, \text { long }\end{array}$ & 22.0 & 350 & 365 & 0.09 & 0.09 \\
\hline
\end{tabular}



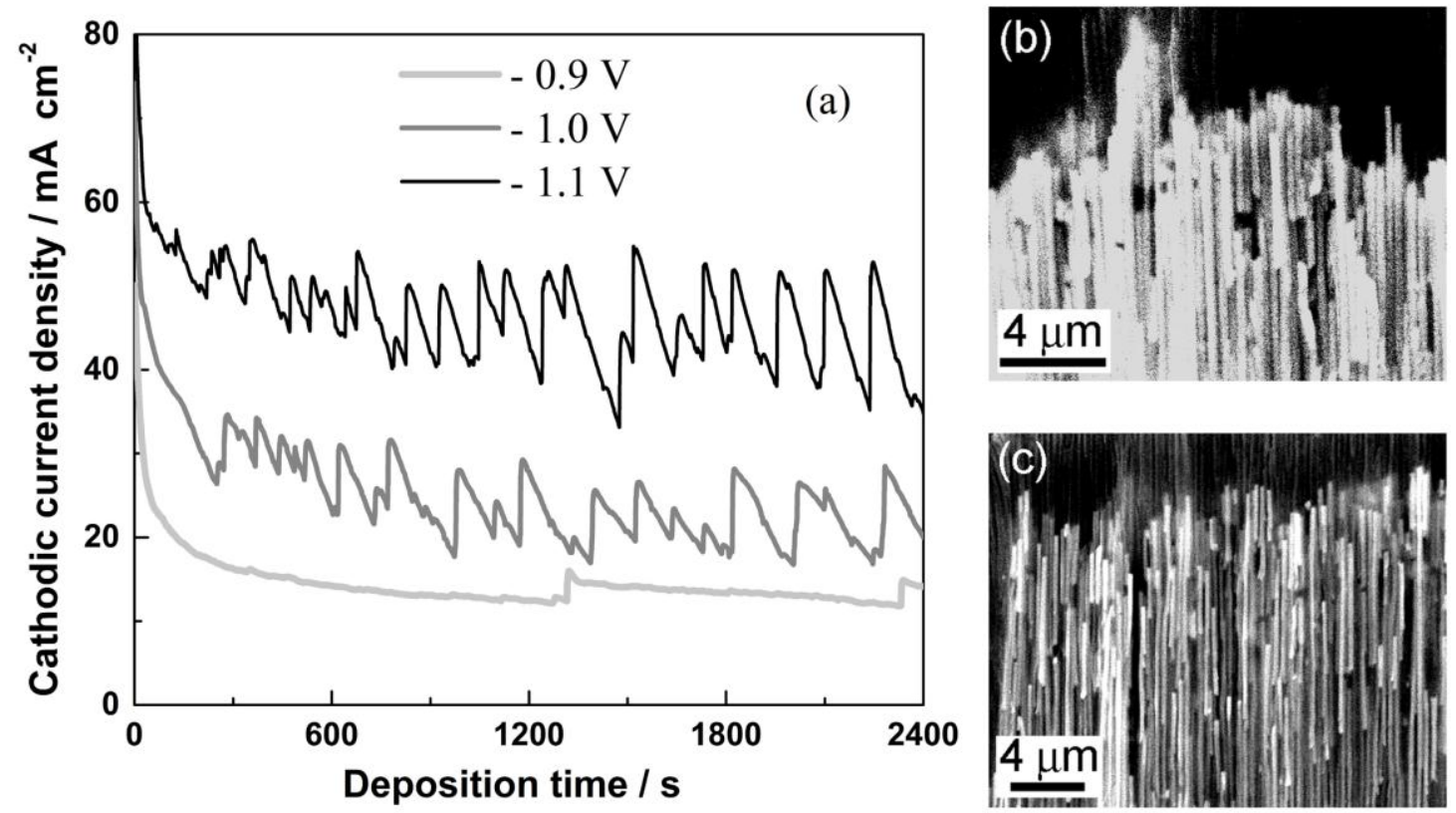

Figure 1

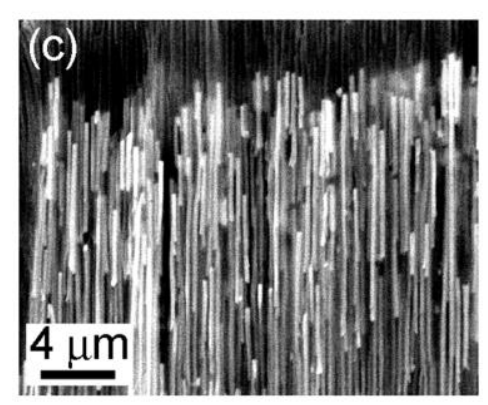




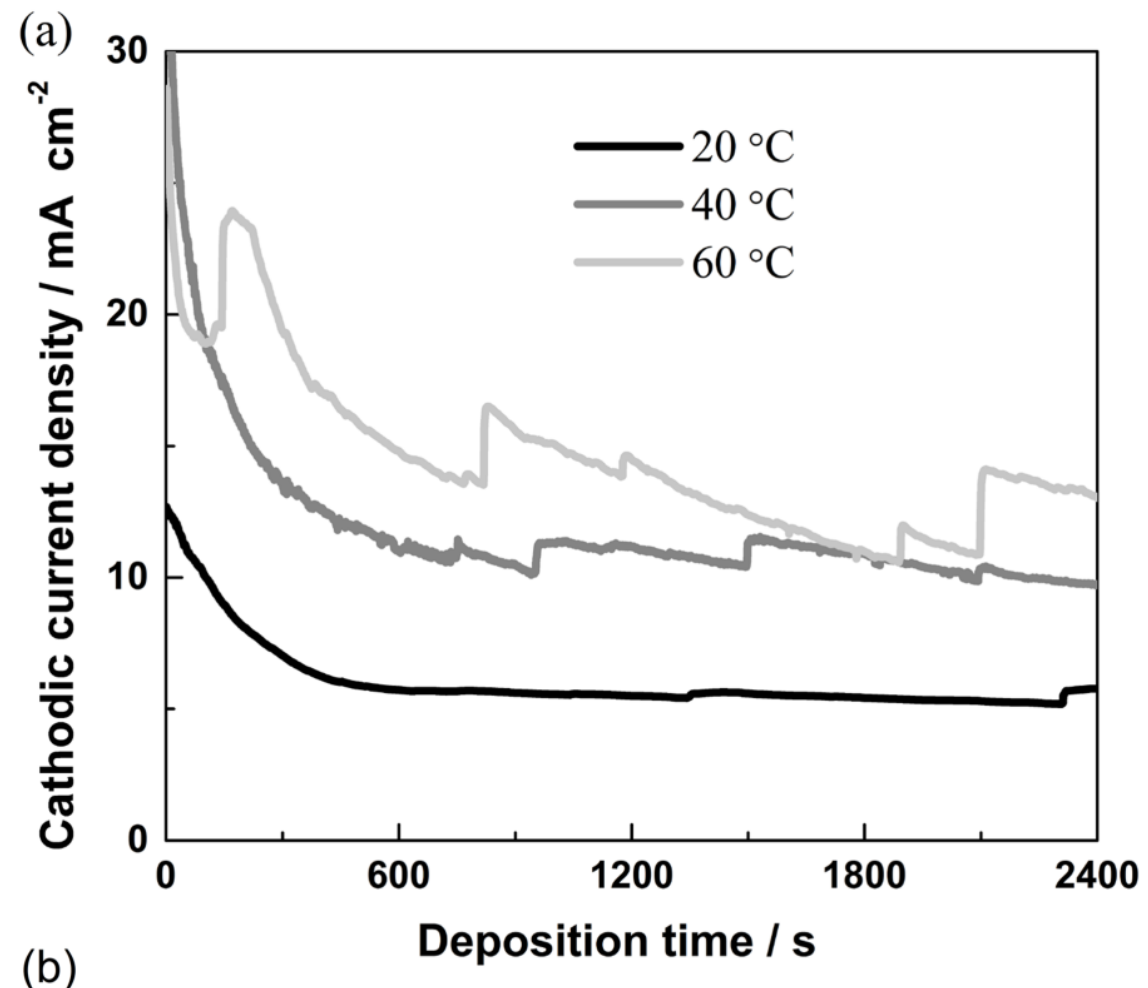

(b)

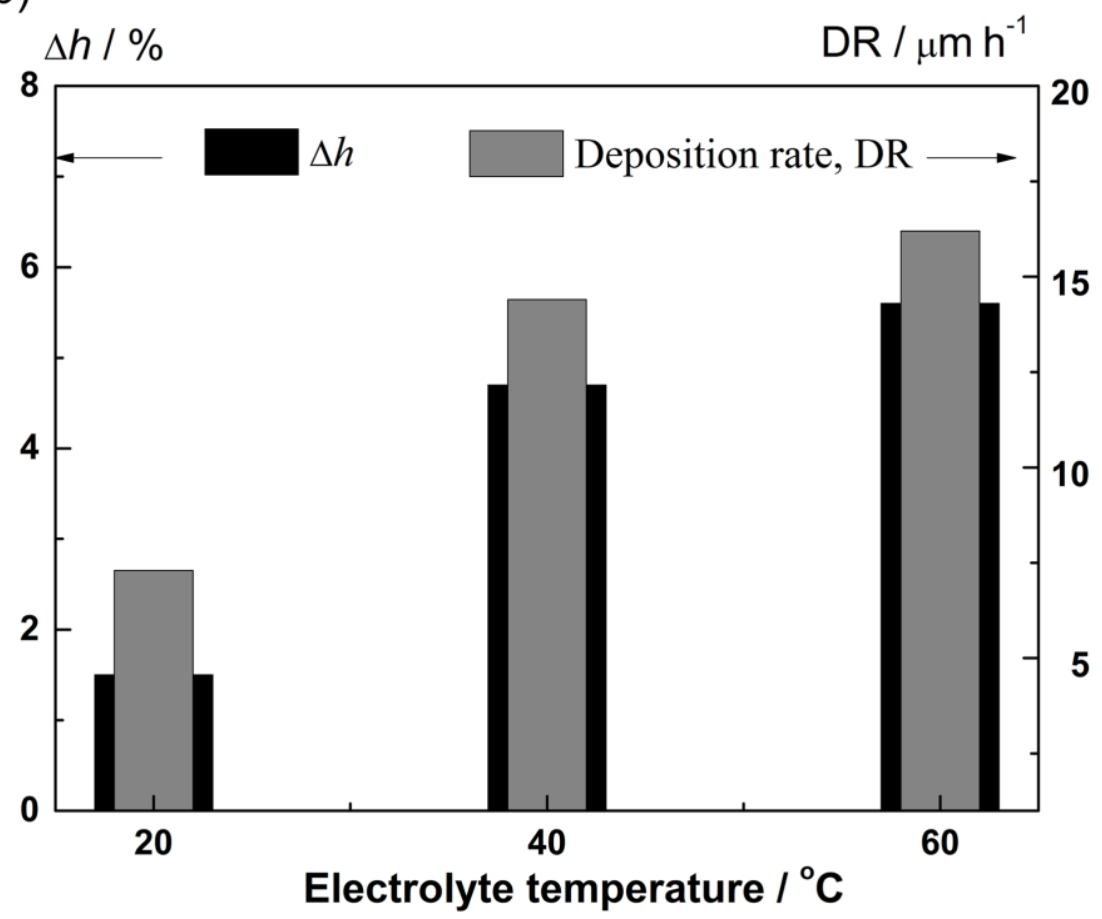

Figure 2 

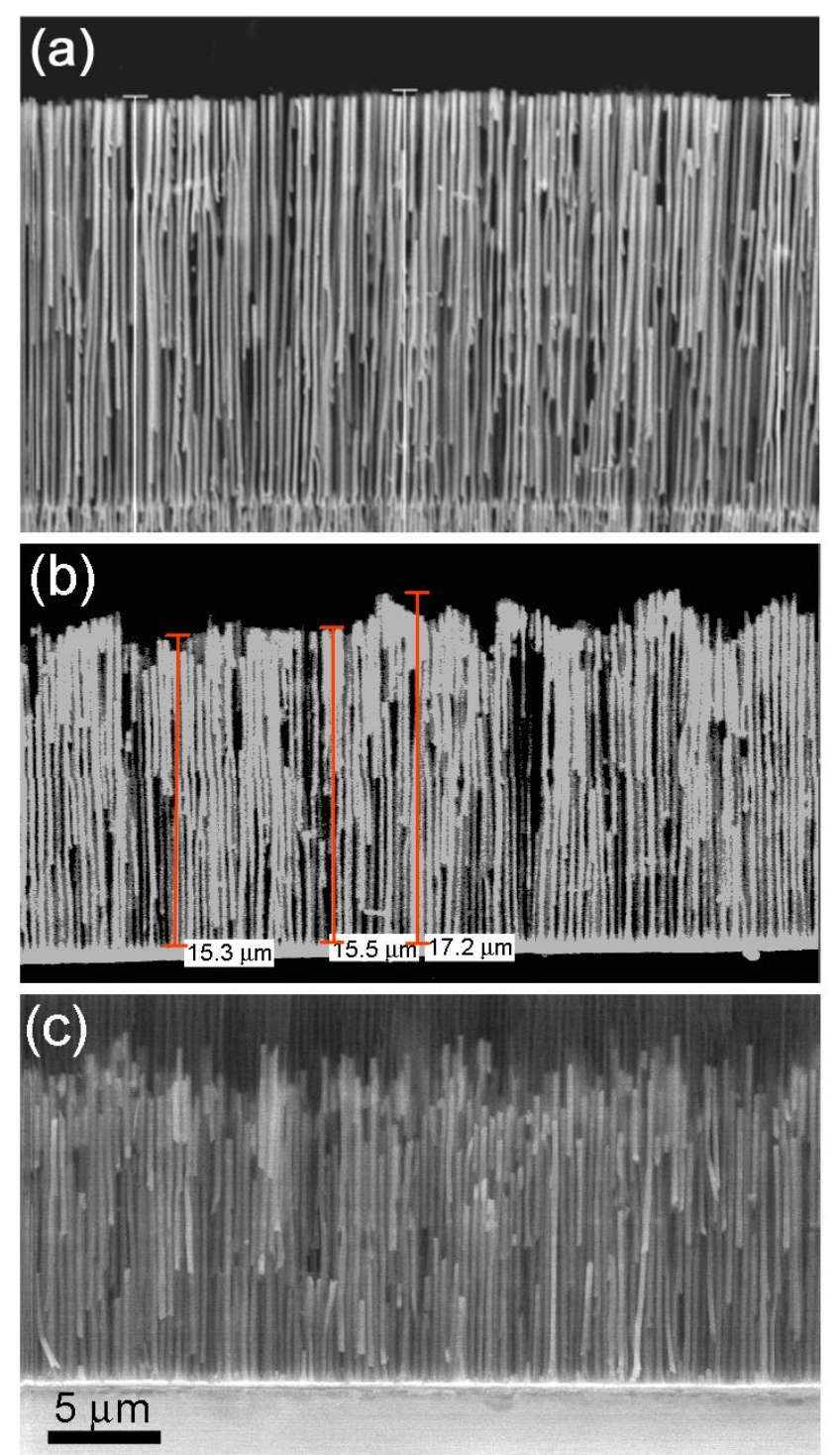

Figure 3 

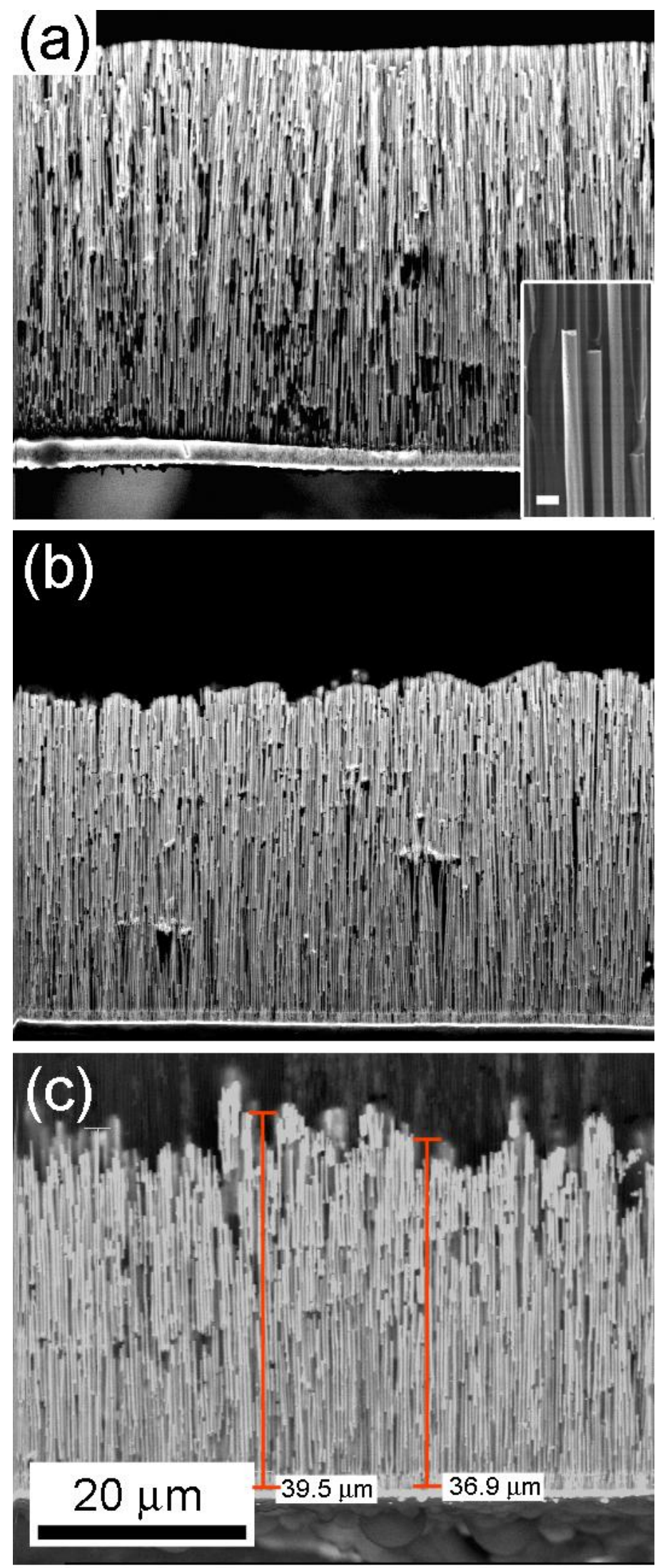

Figure 4 


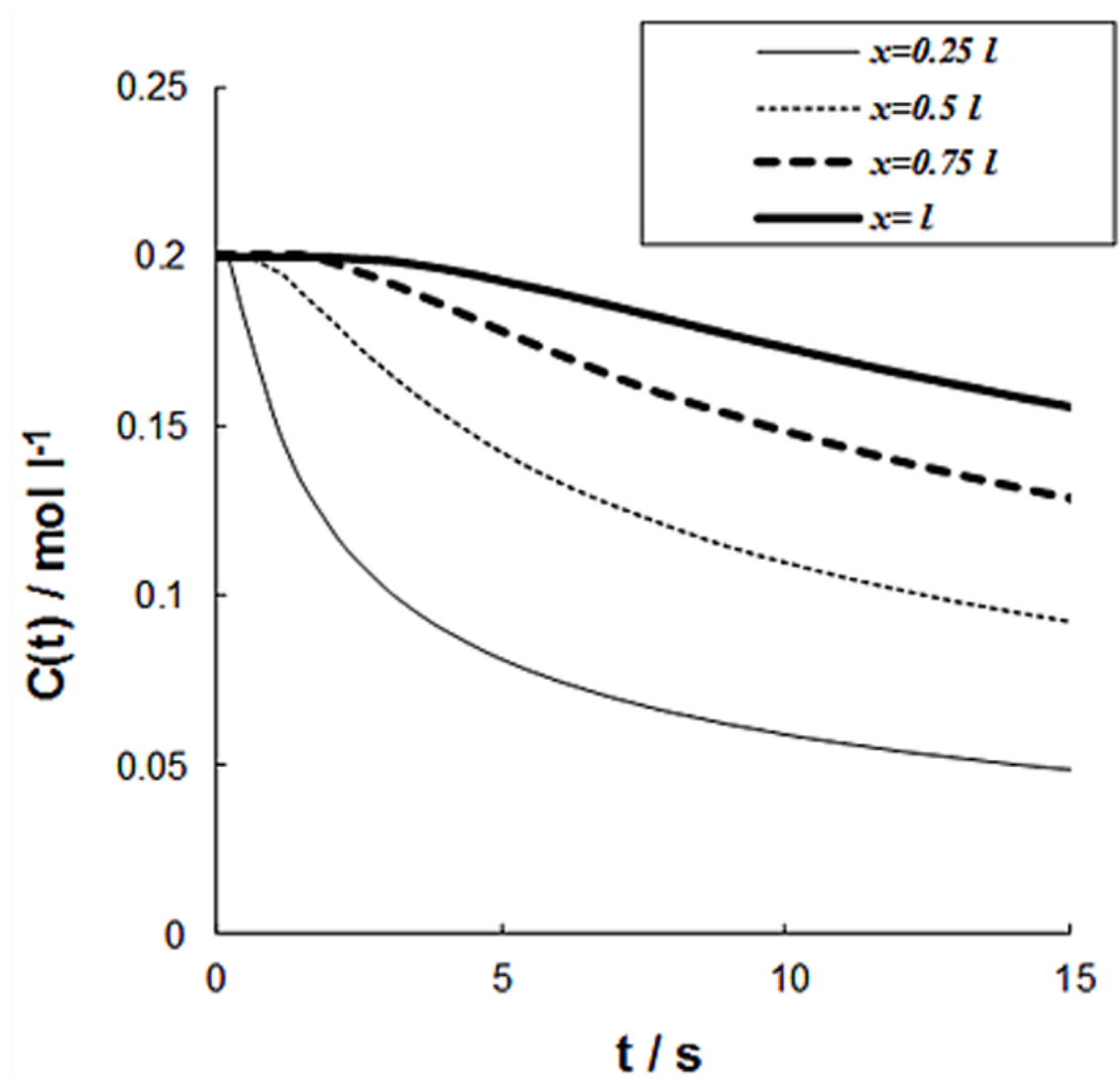

Figure 5 

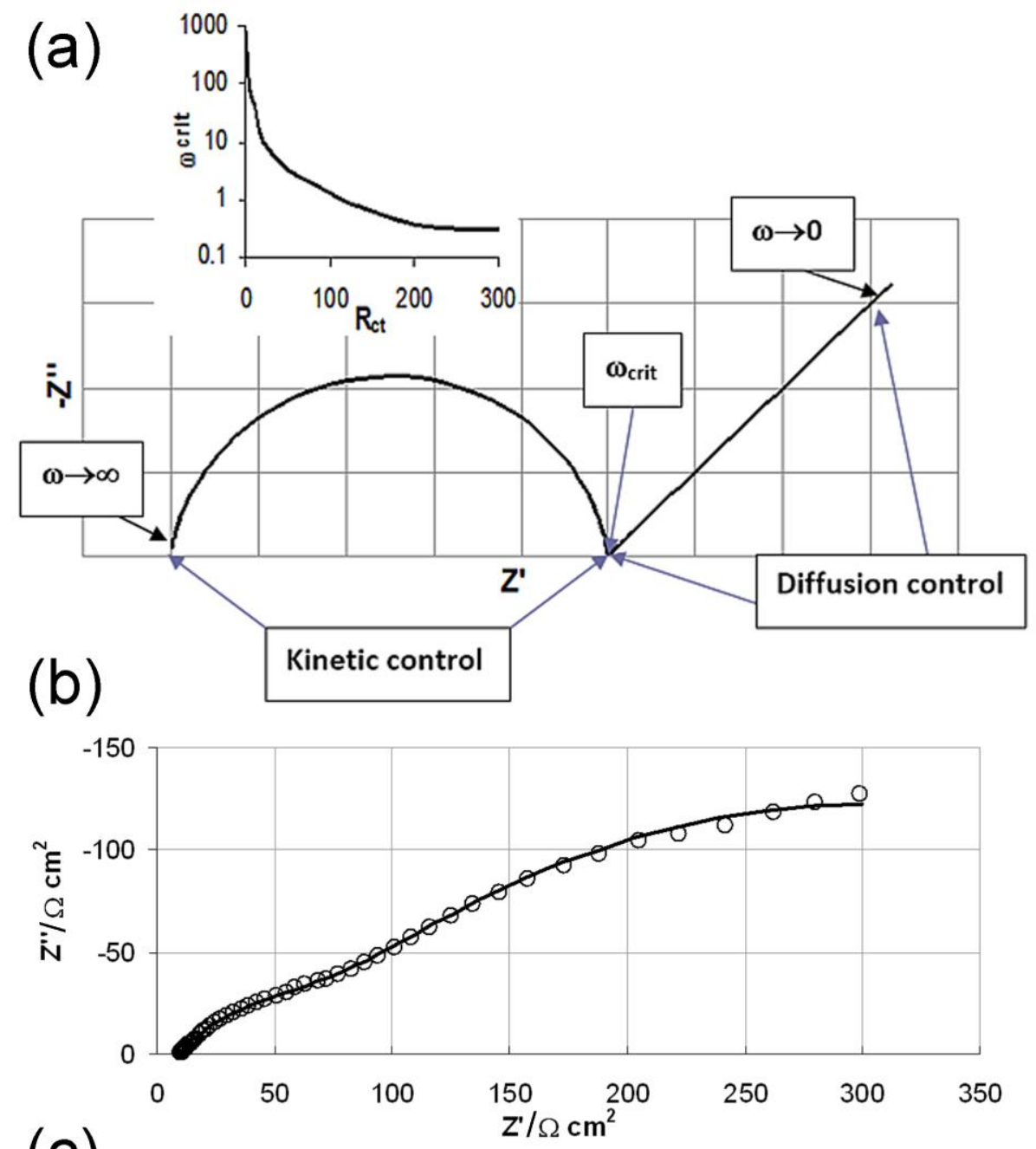

(c)

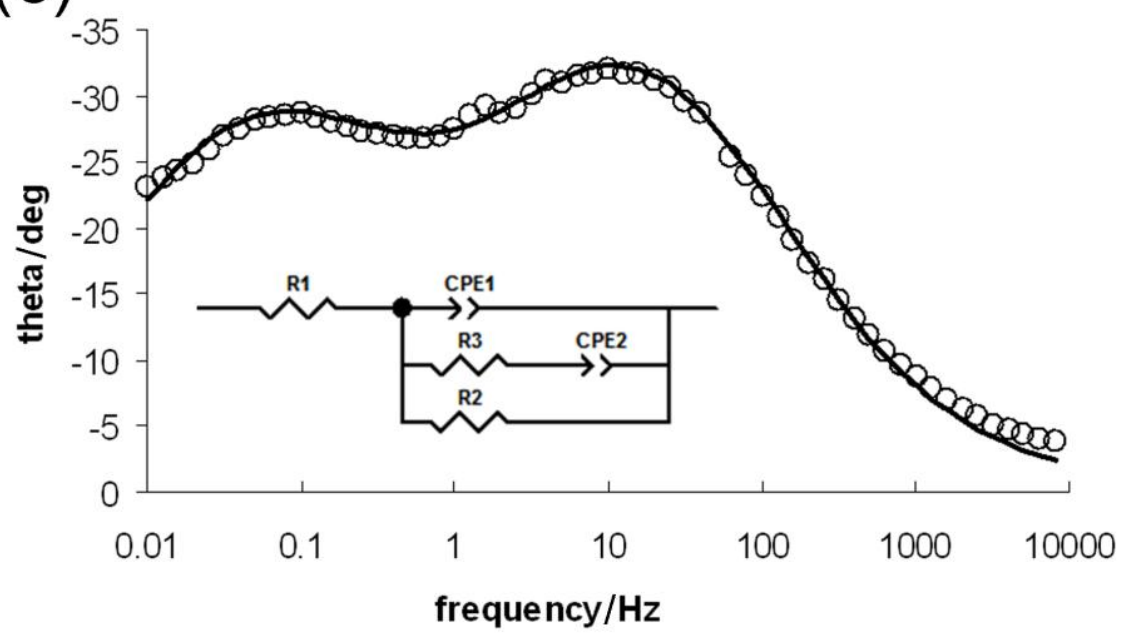

Figure 6 
$15 \mathrm{~mA} \mathrm{~cm}^{-2}$
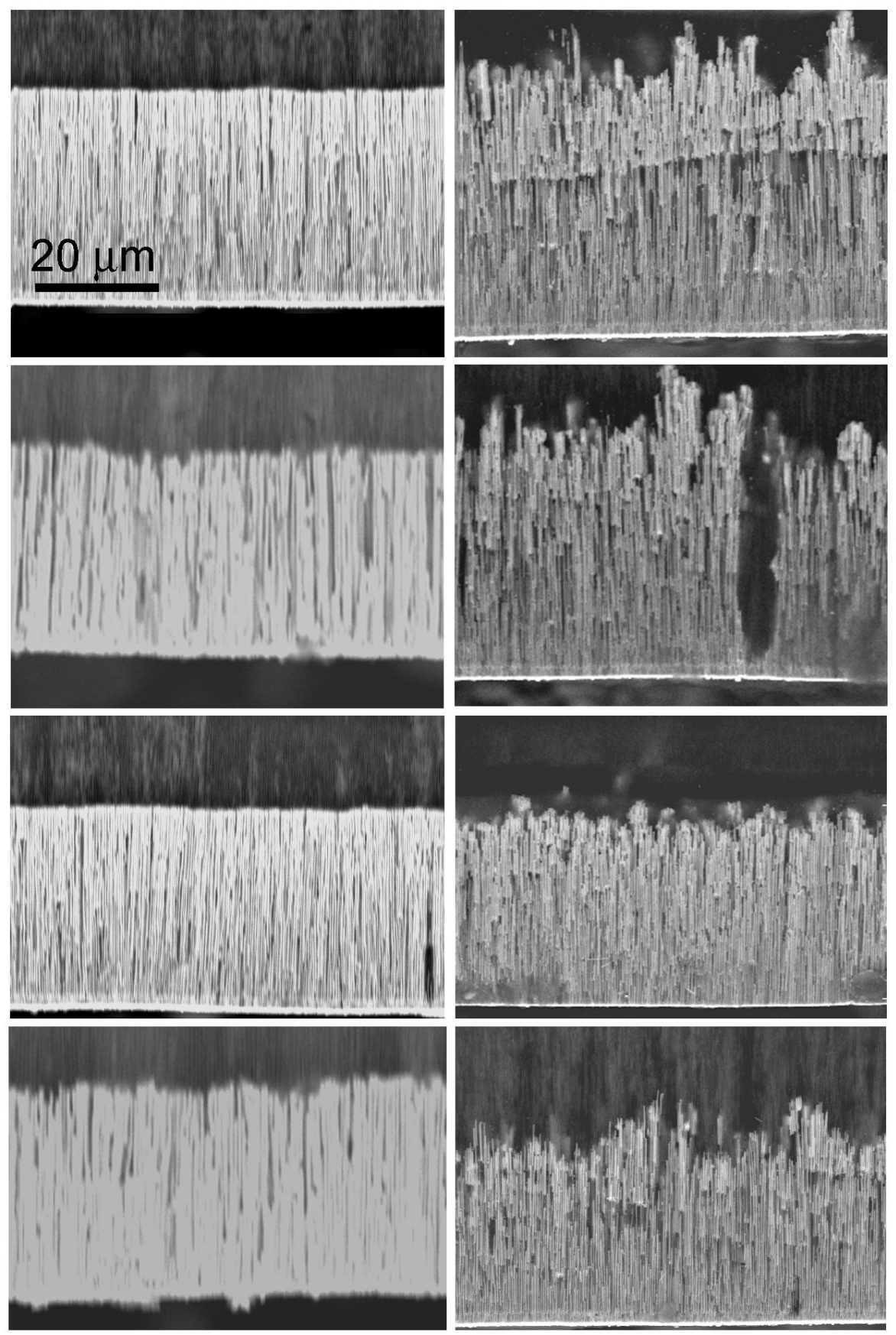

$2000 \mathrm{~ms}$

$20 \mathrm{~ms}$

$200 \mathrm{~ms}$

Figure 7 

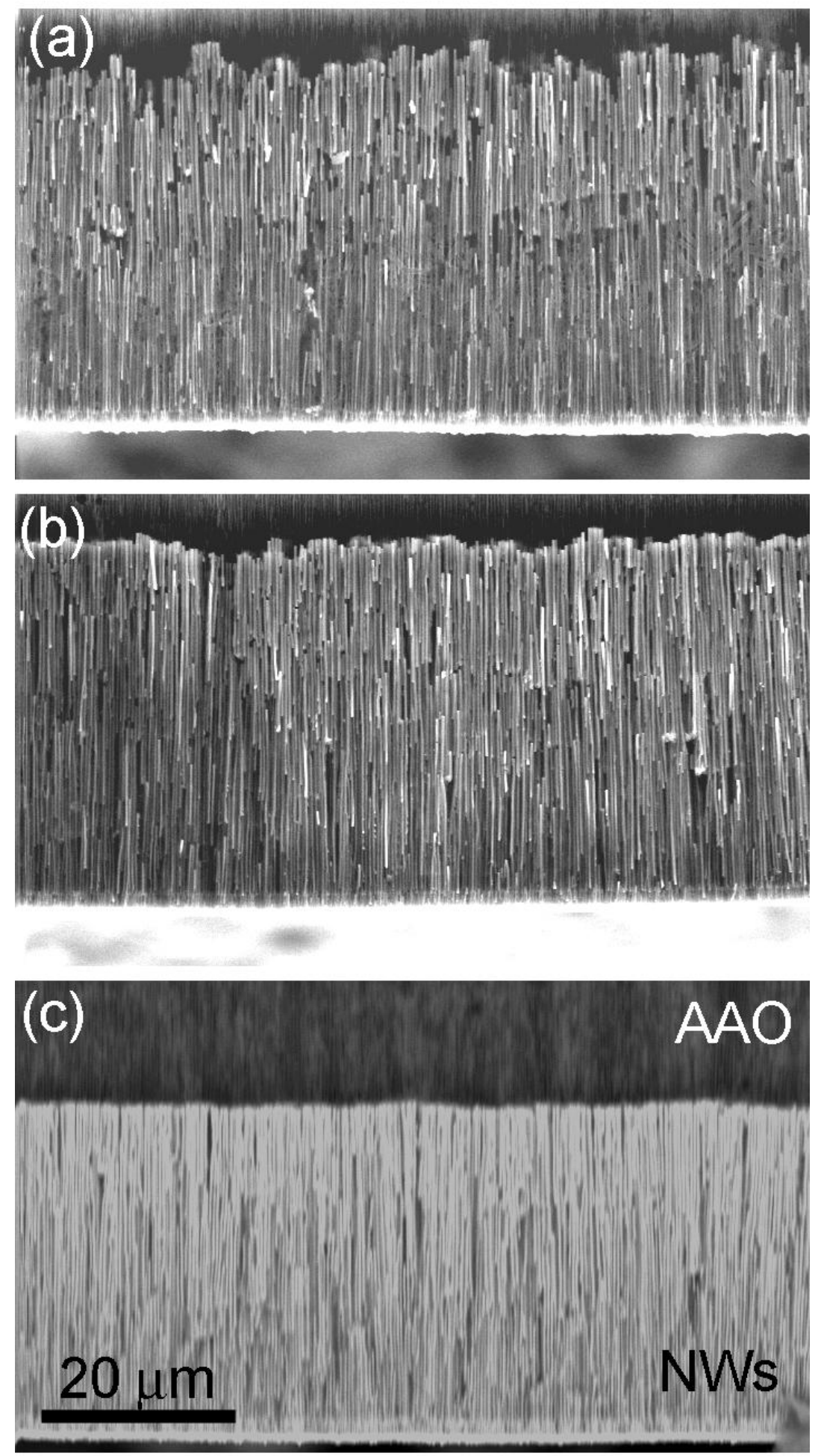

Figure 8 


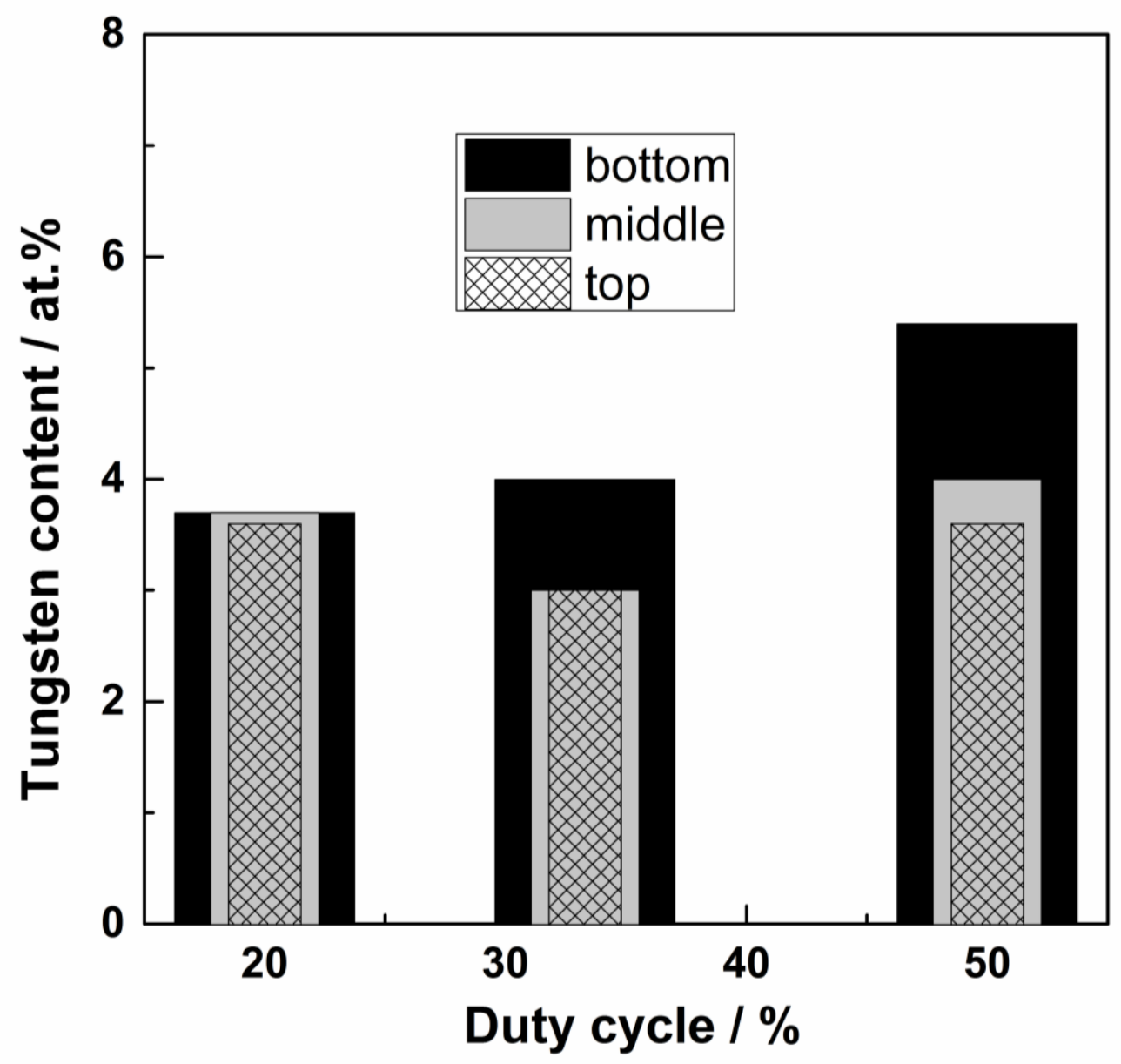

Figure 9 


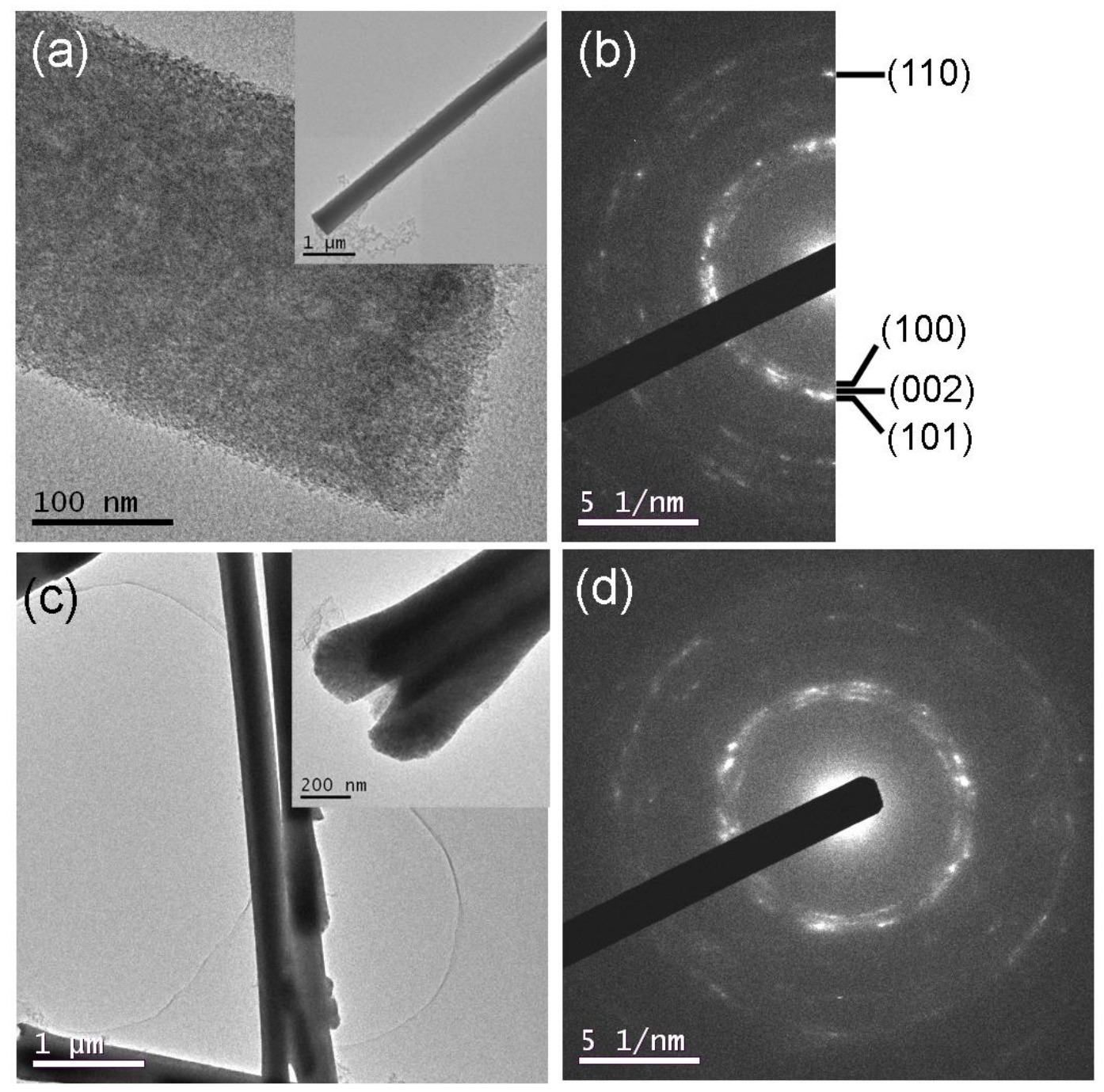

Figure 10 

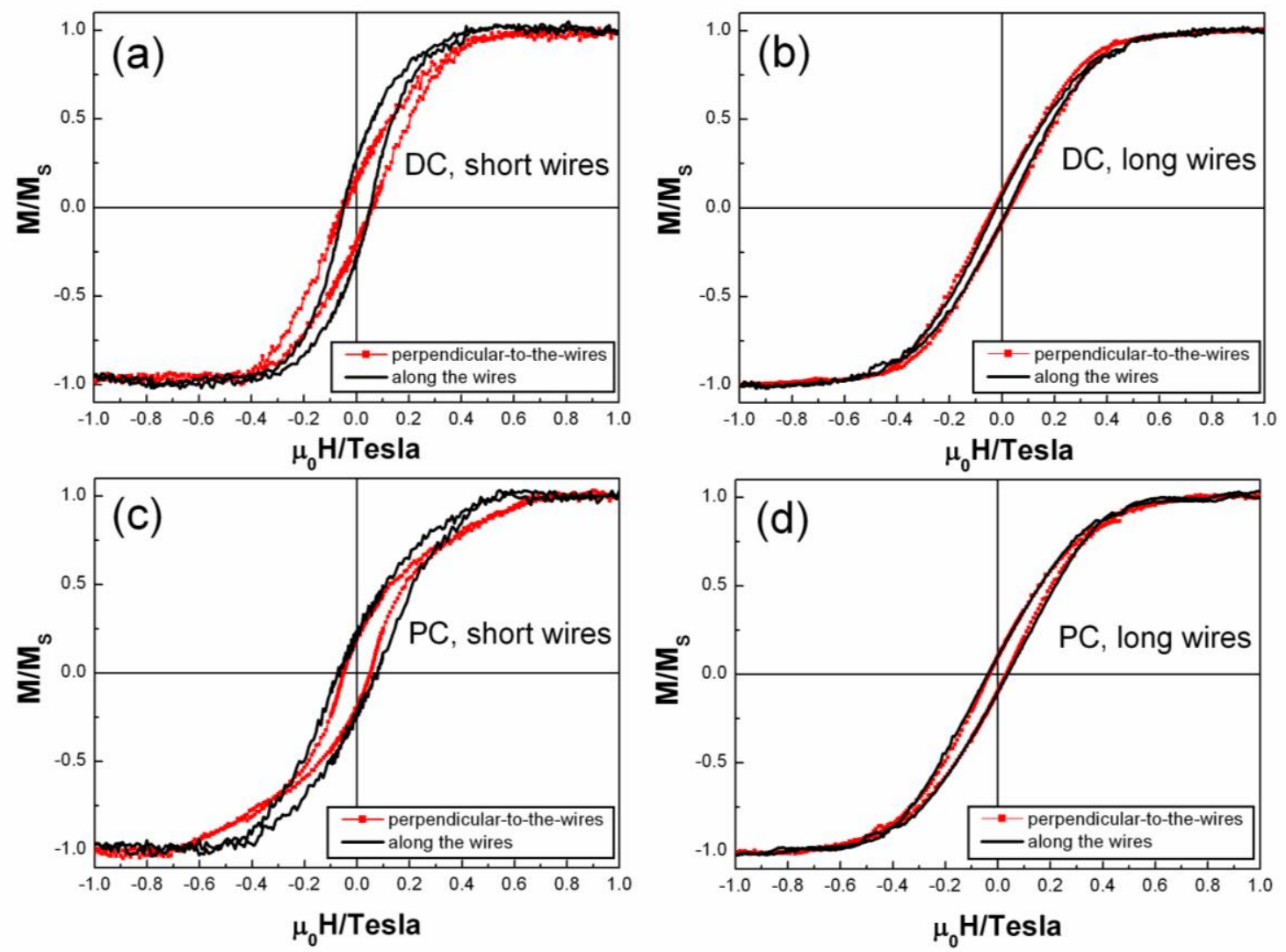

Figure 11 